\title{
Human-Restricted Bacterial Pathogens Block Shedding of Epithelial Cells by Stimulating Integrin Activation
}

\section{Petra Muenzner, ${ }^{1}$ Verena Bachmann, ${ }^{1}$ Wolfgang Zimmermann, ${ }^{2}$ Jochen Hentschel, ${ }^{1,3}$ Christof R. Hauck ${ }^{1,4 *}$}

Colonization of mucosal surfaces is the key initial step in most bacterial infections. One mechanism protecting the mucosa is the rapid shedding of epithelial cells, also termed exfoliation, but it is unclear how pathogens counteract this process. We found that carcinoembryonic antigen (CEA)-binding bacteria colonized the urogenital tract of CEA transgenic mice, but not of wild-type mice, by suppressing exfoliation of mucosal cells. CEA binding triggered de novo expression of the transforming growth factor receptor CD105, changing focal adhesion composition and activating $\beta_{1}$ integrins. This manipulation of integrin inside-out signaling promotes efficient mucosal colonization and represents a potential target to prevent or cure bacterial infections.

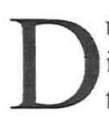
uring colonization of mucosal surfaces, incoming microbes must cope with multiple host defenses (I). One protective mechanism of the mucosa in stratified and squamous tissues is the accelerated turnover and shedding of superficial epithelial cells, also referred to as exfoliation (2-4). Although in vitro studies have suggested that microbes modulate cell detachment $(5,6)$, it is currently unknown how successful mucosal pathogens deal with the exfoliation response in vivo. Neisseria gonorrhoeae, a Gram-negative microorganism, causes one of the most common sexually transmitted diseases worldwide (7). Even though these bacteria can induce the exfoliation of host cells upon contact $(8-11)$, they are able to establish themselves on virtually every mucosal surface of the human body.

To investigate how gonococci manage to colonize the urogenital mucosa efficiently, we performed vaginal infection of female mice (I2). In line with the innate capacity of epithelial cells to respond to this bacterial challenge, $N$. gonorrhoeae triggered detachment of superficial epithelial cells within 20 hours (Fig. 1A) and only small numbers of gonococci could be re-isolated from wild-type mice (Fig. 1B). Gonococci are adapted to humans as their sole natural host. One of the host-specific virulence traits that gonococci share with other specialized mucosal colonizers, including Haemophilus influenzae, Moraxella catarrhalis, and $N$. meningitidis, is the ability to recognize hu-

${ }^{1}$ Lehrstuhl Zellbiologie, Fachbereich Biologie, Universität Konstanz, 78457 Konstanz, Germany. ${ }^{2}$ Tumor Immunology Laboratory, LIFE Center, Ludwig-Maximilians-Universität München, 81377 München, Germany. ${ }^{3}$ EM Service, Fachbereich Biologie, Universität Konstanz, 78457 Konstanz, Germany. ${ }^{4}$ Konstanz Research School Chemical Biology, Universität Konstanz, 78457 Konstanz, Germany.

*To whom correspondence should be addressed. E-mail: christof.hauck@uni-konstanz.de 
man carcinoembryonic antigen-related cell adhesion molecules (CEACAMs) $(13,14)$. To engage CEACAMs, gonococci use outer membrane adhesins of the colony opacity-associated (Opa) protein family [Opa ${ }_{C E A}$; for a review see (15)]. Bacterial engagement of human CEACAMs can block detachment of infected epithelial cells in vitro, which suggests that gonococci and other CEACAM-binding bacteria might use CEACAMs to modulate exfoliation (5).

To test the hypothesis that CEACAM engagement by bacteria attenuates epithelial exfoliation and promotes mucosal colonization, we used a humanized mouse model of gonococcal urogenital tract infection: CEA transgenic (CEAtg) mice harboring the human CEACAM5 gene (16). In CEAtg mice, CEA can be detected on mucosal surfaces including the female urogenital tract (fig. S1A). Wild-type and CEAtg female mice were

Fig. 1. CEA binding facilitates mucosal colonization and blocks exfoliation of superficial epithelial cells. (A) Female wild-type mice were infected for 24 hours with piliated, non-opaque gonococci, or remained uninfected. Genital tracts were excised, fixed, and processed for SEM. Micrographs show the luminal surface of the upper vaginal and cervical region. Exfoliating cells are highlighted by arrows. (B) Schematic representation of the infection protocol used with respect to pretreatment of the mice, vaginal infection, and re-isolation of bacteria ( $\Delta$ indicates the time point of lentiviral transduction). The graph shows colonization of wild-type (O) or CEAtg (O) female mice with the indicated bacterial strains. Each circle reflects the number of bacteria re-isolated from an individual animal ( $n=8$ unless indicated otherwise); data were compiled from six experiments. The dotted line indicates the lower detection limit (40 bacteria per animal). The median for each experimental group of animals is noted; groups were compared against numbers isolated from $\mathrm{Ngo}$ Opa $\mathrm{PEA}_{\text {-infected CEAtg }}$ mice by two-tailed Mann-Whitney U-test $\left({ }^{*} P<\right.$ 0.001). (C) Genital tracts infected as in (B) were excised and processed for SEM. Pictures (at two different magnifications, as indicated by the scale bars) show the luminal surface of the upper vaginal and cervical regions. Inset shows adherent bacteria (black arrowhead). (D) Quantification of exfoliating epithelial cells. Bars represent mean \pm SD of exfoliating cells in an area of about $0.075 \mathrm{~mm}^{2}$ $(n=24)$; groups were compared against uninfected mice by one-tailed Mann-Whitney U-test $\left({ }^{*} P<\right.$ 0.01). vaginally infected with $10^{8}$ bacteria, and colonizing bacteria were recovered by urogenital swabs 24 hours later. Consistent with the initial observations, only a few gonococci could be re-isolated from wild-type mice irrespective of the bacterial phenotype, whereas the numbers of gonococci expressing Opa $\mathrm{CEA}_{\mathrm{C}}$ proteins recovered from CEAtg mice were higher by a factor of about 50 to 100 (Fig. 1B). The Opa $\mathrm{CEA}_{\mathrm{A}}$ protein expression by reisolated bacteria was unaltered (fig. S1B). Some re-isolates expressed additional Opa proteins, further pointing to an in vivo advantage of opaque phenotypes. Isogenic gonococcal strains lacking Opa protein expression $\left(\mathrm{Opa}^{-}\right)$, strains expressing type IV pili $\left(\mathrm{Opa}^{-} / \mathrm{P}^{+}\right)$, or strains expressing a heparan sulfate proteoglycan-binding Opa protein $\left(\mathrm{Opa}_{\mathrm{HSPG}}\right)(17,18)$ could not be isolated in increased numbers from CEAtg mice (Fig. 1B). Scanning electron microscopy (SEM) of the upper vaginal tract revealed massive exfoliation of the superficial epithelial layer in wild-type animals infected with Opa $\mathrm{CEA}_{\mathrm{C}}$-expressing $N$. gonorrhoeae (Ngo Opa ${ }_{\text {CFA }}$ ) as well as in CEAtg animals infected with non-CEACAM-binding gonococci (Fig. IC). Vast exfoliation was already evident at low magnification; at higher magnification, the detachment of multiple superficial epithelial cells was apparent (Fig. 1C). In contrast, exfoliation was not elevated in CEAtg mice infected with Ngo Opa $a_{\text {CEA }}$ (Fig. 1, C and D). Thus, the Opa $\mathrm{CLA}^{-}$ CEA interaction suppresses exfoliation and facilitates mucosal colonization.

In the urogenital tract of infected mice, gonococci colocalized with CEA-positive cells on the vaginal surface of CEAtg mice (Fig. 2, A and B) Gonococci lacking Opa protein expression (Ngo Opa ) were rarely detected on the mucosal surface of CEAtg mice, and gonococci were absent in
A

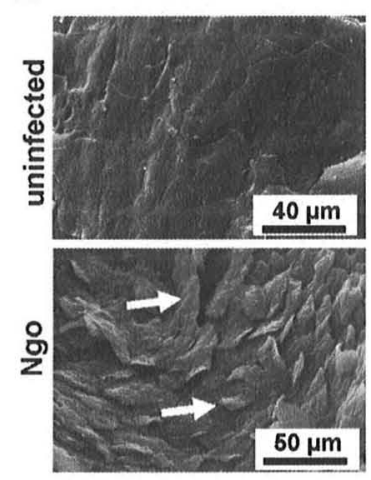

\section{B}
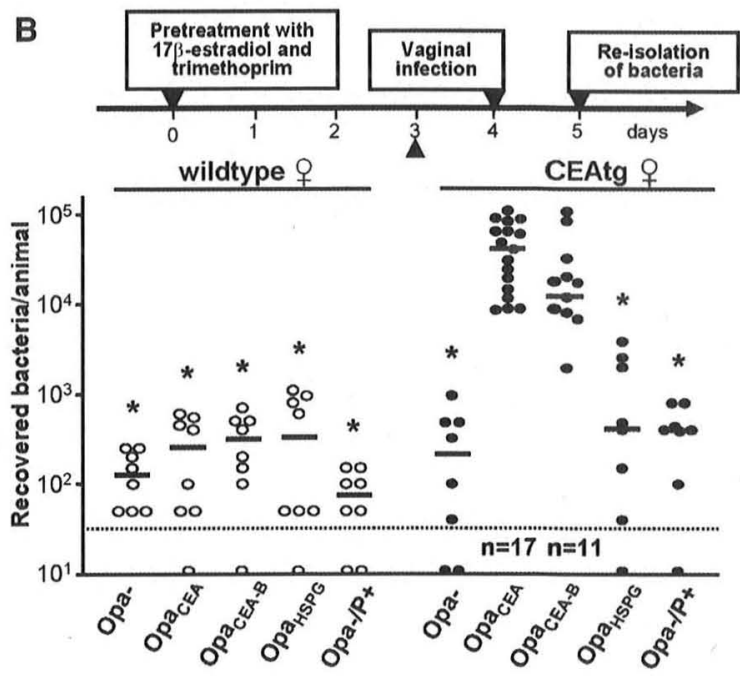

C
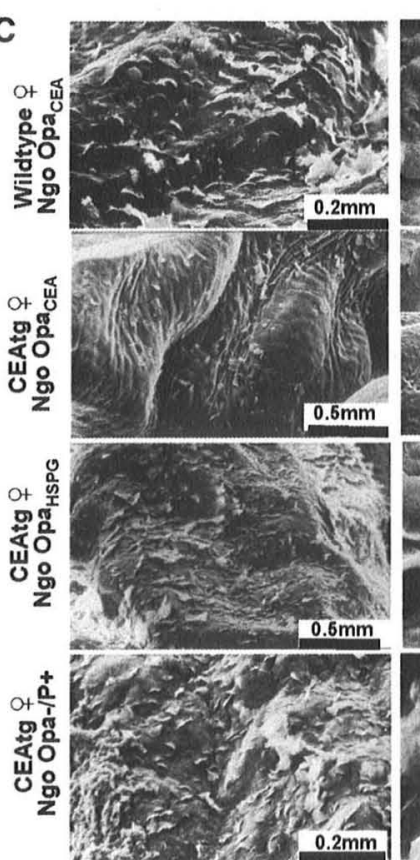
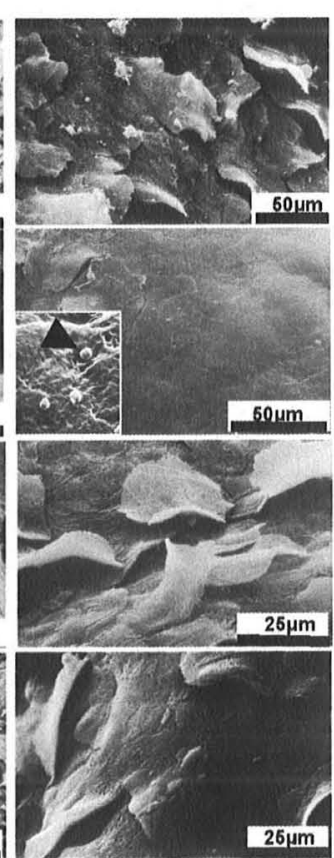

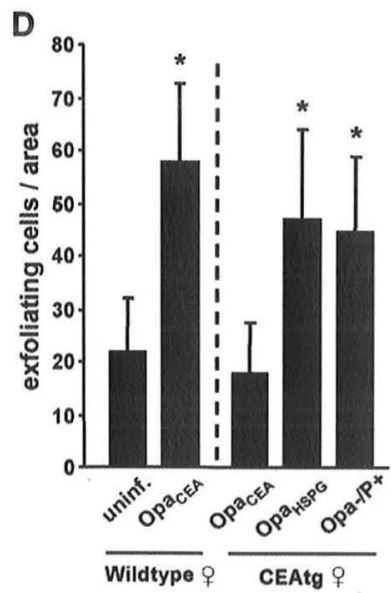


Fig. 2. CEA engagement by bacteria triggers CD105 expression in vivo. (A) Genital tracts from wild-type or CEAtg mice infected for 24 hours with Opa ${ }_{\text {CEA }}$ expressing ( $\mathrm{Ngo}^{-} \mathrm{Paa}_{\mathrm{CEA}}$ ) or non-opaque ( $\mathrm{Ngo} \mathrm{Opa}^{-}$) gonococci were excised, and cryosections were costained with antibodies against $N$. gonorrhoeae (green) and CEA (red). Cell nuclei were visualized by Hoechst (blue). Ngo Opa $\mathrm{OCF}_{\mathrm{CEA}}$ bound to the CEA-positive mucosal surface of CEAtg mice are indicated by arrowheads. (B) Quantification of cell-associated bacteria in CEAtg mice. Bars represent the mean number \pm SD of bacteria associated with 100 cells $(n=3)$. (C) Cryosections as in (A) were costained with antibodies against $N$. gonorrhoeae (green), a rat monoclonal antibody against murine CD105 (red), and nuclei (blue). CD105 expression (arrowheads) on the mucosal surface of CEAtg mice is seen in the vicinity of cell-associated $\mathrm{Ngo} \mathrm{Opa}_{\text {CEA }}$.
A
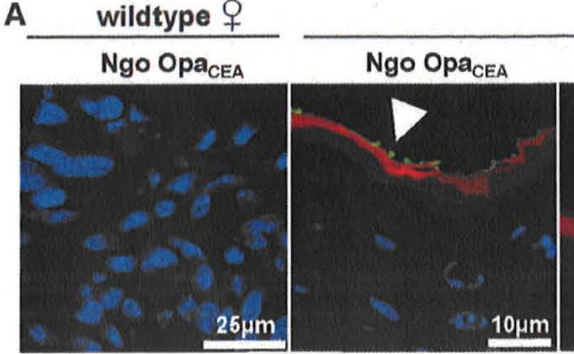

CEAtg $O$

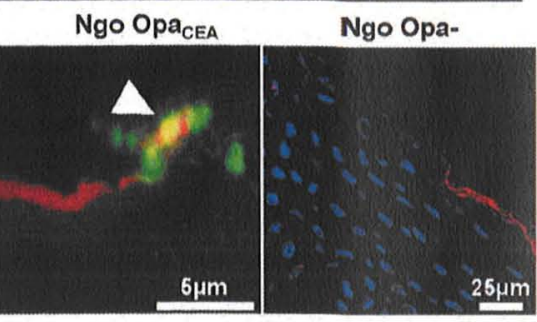

B

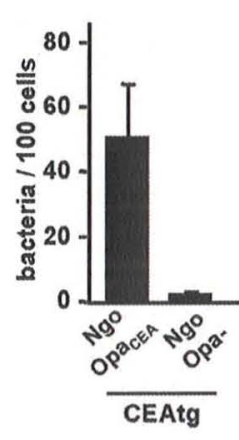

C

CD105

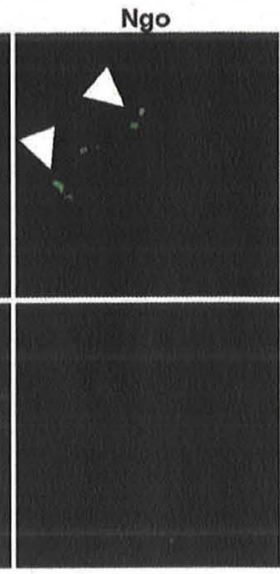

overlay

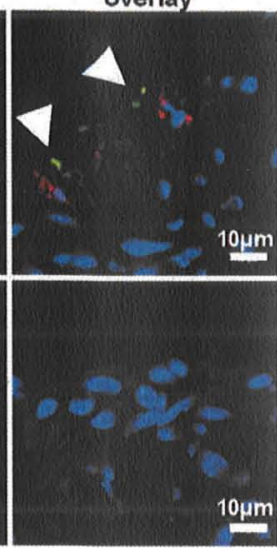

A

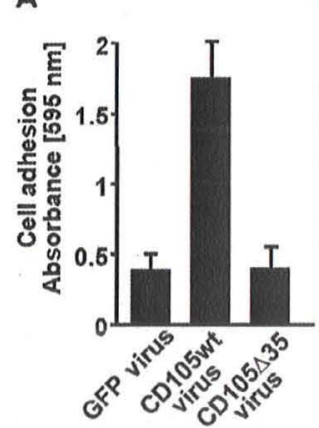

C
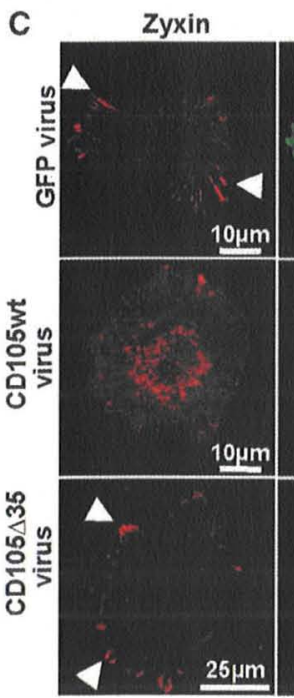

B
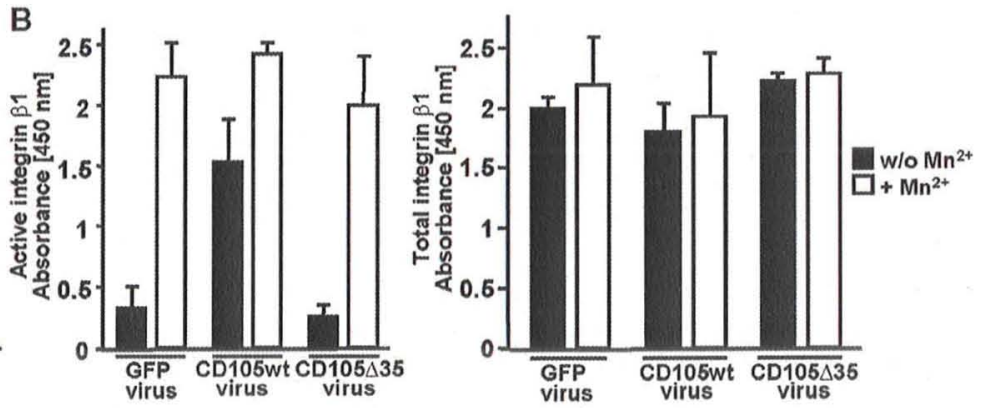

GFP

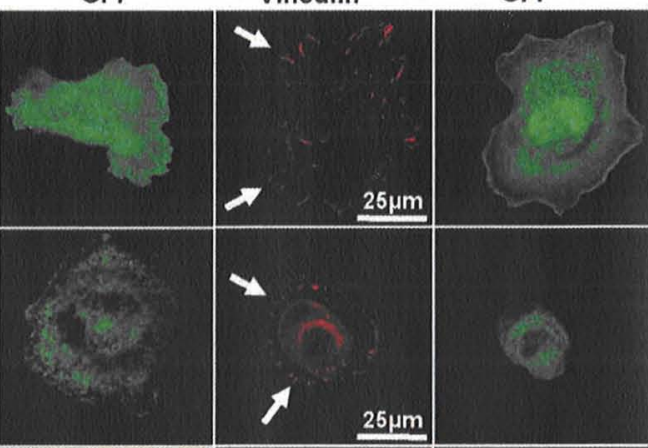

D

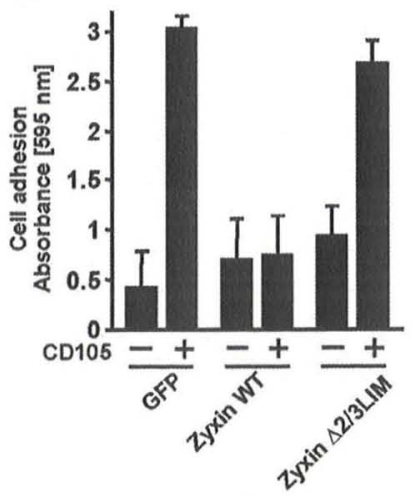

Fig. 3. CD105 enhances integrin activity by delocalization of zyxin. (A) Human vaginal epithelial cells (hVECs) were transduced with lentivirus encoding GFP, wild-type CD105 (CD105wt), or CD105 135 . Cells were used in adhesion assays, and adherent cells were quantified after crystal violet staining. Bars represent mean \pm SD of five wells. (B) Cells transduced as in (A) were replated onto collagen and stimulated with $\mathrm{Mn}^{2+}$ (a global activator of integrin activity), or remained unstimulated, before analysis with an activationspecific integrin $\beta_{1}$ antibody (done 9EG7; active integrin $\beta_{1}$ ) or an activation-independent integrin $\beta_{1}$ antibody (clone AllB2; total integrin $\beta_{1}$ ). Bars represent the mean $\pm S D$ of five wells of a representative experiment repeated twice with similar results. (C) hVECS were transduced as in (A), and parallel samples were stained for zyxin or vinculin. Small arrowheads highlight zyxin at focal adhesions, which is missing in CD105wtexpressing cells. Vinculin-positive focal adhesions (arrows) are found in all cells. (D) HeLa cells were transfected with vectors encoding GFP, GFP-zyxin, or GFP-zyxin $\triangle 2 / 3 \mathrm{LIM}$, in the presence or absence of CD105. Cells were used in adhesion assays as in (A). Bars represent mean \pm SD of five wells. 
wild-type mice, even if infected with Ngo Opa $\mathrm{CEA}$ (Fig. 2, A and B). How can the engagement of apically expressed CEA lead to reduced detachment of infected cells? CEACAM-binding by bacteria triggers de novo expression of CD105, a member of the transforming growth factor- $\beta 1$ receptor (TGF $\beta 1 R$ ) family, which promotes cell-matrix adhesion and blocks cell migration $(5,19)$. Indeed, CEA engagement by $\mathrm{Ngo} \mathrm{Opa}_{\mathrm{CEA}}$ in vitro stimulated the expression of $\mathrm{CD} 105$ and increased the adhesiveness of infected cells to several extracellular matrix proteins (fig. S2). Moreover, CEACAMbinding bacteria, but not Opa ${ }^{-}$bacteria, triggered de novo expression of CD105 by vaginal epithelial cells of CEAtg mice in vivo (Fig. 2C and fig. S3). CD105, a well-characterized endothelial marker, was present on small blood vessels in the submucosa of wild-type (fig. S4) and CEAtg mice, but was absent from uninfected vaginal epithelial cells (Fig. 2C and fig. S3). Thus, CD105 expression in response to CEA stimulation by gonococci could be responsible for enhanced matrix adhesion of infected epithelial cells and the suppression of exfoliation.

Cell adhesion to the extracellular matrix is mediated by integrins, and TGF $\beta 1$ Rs influence integrin expression (20). However, CD105, a type III nonsignaling member of the TGF $\beta 1 R$ family, does not increase the amount of integrin $\beta_{1}$ or integrin-associated cytoplasmic proteins $(19,21)$. In HeLa cells and 293 cells, CD105 promoted cell adhesion by stimulating integrin inside-out signaling, leading to enhanced integrin activity (fig. S5). The adhesion-promoting activ- ity of CD105 was located in the cytoplasmic domain of CD105, which was necessary (fig. S6) and sufficient (fig. S7) for this process. In line with these results, transduction with wild-type CD105 (CD105wt), but not with CD105 carrying a 35-amino acid truncation of the cytoplasmic domain $(\mathrm{CD} 105 \Delta 35)$, enhanced cell-matrix adhesion and increased integrin activity of human vaginal epithelial cells (hVECs) (Fig. 3, A and B). One of the cellular factors that associate with the cytoplasmic part of CD105 is zyxin, a focal adhesion protein that binds $C D 105$ via three LIM (Lin-11 Isl-1 Mec-3) domains (19). Zyxin-deficient fibroblasts show enhanced integrin activity and matrix adhesion (22), which was not further stimulated by CD105 expression (fig. S8). The dependence of CD105-stimulated integrin activity on zyxin, which by itself acts as a negative regulator of cell adhesion, could be explained by a scenario where de novo expression of CD105 interferes with zyxin's inhibitory function at focal adhesion sites. In hVECs, which express zyxin but not CD105 (fig. S9), zyxin was located at peripheral contact sites (Fig. 3C). However, upon expression of CD105wt, but not CD105 $\triangle 35$, zyxin was delocalized (Fig. $3 \mathrm{C}$ and fig. S9). In contrast, the localization of the focal adhesion protein vinculin was unaltered (Fig. 3C). Increasing the cellular pool of zyxin by overexpression, but not by expression of a zyxin mutant lacking the second and third LIM domains (zyxin $\triangle 2 / 3$ LIM), abrogated the increased adhesion of CD105-transfected cells (Fig. 3D). Thus, CD105-mediated delocalization of endogenous zyxin positively influences integrin activity and could be responsible for the enhanced cell adhesion and the suppression of epithelial exfoliation triggered by CEACAM-binding bacteria.

To test a causal relationship among CEACAMdependent up-regulation of $\mathrm{CD} 105$, suppression of epithelial exfoliation, and improved colonization of the urogenital mucosa by pathogenic gonococci, we generated a GFP (green fluorescent protein) and short hairpin RNA-encoding lentivirus targeting murine CD105 (shCD105) (fig. S10). In vivo, vaginal application of shCD105 or a GFP control virus resulted in strong expression of GFP in the superficial epithelial layer (fig. S10). To investigate the role of $\mathrm{CD} 105$ expression for mucosal colonization in vivo, we applied shCD105 or GFP control virus 24 hours before vaginal infection of mice. Application of shCD105 virus suppressed the ability of $\mathrm{Ngo} \mathrm{Opa}_{\mathrm{CEA}}$ to colonize the urogenital tract of CEAtg mice, whereas the GFP control virus had no effect (Fig. 4A). Moreover, SEM of the mucosa revealed that transduction with shCD105 virus reversed the phenotype of the infected epithelial cells and allowed pronounced exfoliation in response to infection with CEACAM-binding bacteria (Fig. 4, B and C). In CEAtg mice treated with the GFP control virus, Ngo Opa ${ }_{\mathrm{CFA}}$ were still able to block exfoliation (Fig. 4, B and C). Thus, up-regulation of CD105 on mucosal epithelial cells interferes with exfoliation and promotes gonococcal colonization of the urogenital tract. To further confirm the causal relationship between CD105-initiated molecular processes and the suppression of epithelial exfoliation, we transduced CEAtg mice with lentiviral
Fig. 4. Bacterial colonization is blocked by inhibition of CD105 expression in vaginal epithelial cells or by mucosal delivery of zyxin. (A) Female mice were transduced with GFP, shCD105-encoding virus, or left without virus (w/o virus). Twenty-four hours later, animals were infected with $\mathrm{Ngo} \mathrm{Opa}_{\mathrm{CEA}}$, and colonizing gonococci were enumerated the next day. Bars represent the mean $\pm S D$ of bacteria re-isolated from six individual animals. Groups were compared against numbers isolated from untransduced CEAtg mice by one-tailed Mann-Whitney U-test $\left.{ }^{*} P<0.01\right)$. (B) SEM of the mucosal epithelium of mice transduced and infected as indicated. Exfoliating epithelial cells are marked by arrows; cell-associated bacteria are indicated by arrowheads. (C) Quantification of exfoliating epithelial cells as in Fig. 1D; groups were compared against GFP-transduced wild-type mice by one-tailed Mann-Whitney U-test $\left({ }^{*} P<0.01\right)$. (D and $\left.\mathbf{E}\right)$ Wild-type or CEAtg mice were transduced with lentiviral particles encoding GFP, GFP-zyxin, or GFP-zyxin $\triangle 2 / 3 L I M$, or left without virus (w/o virus) before infection with Ngo Opa $_{\text {CEA }}$. Colonizing gonococci in (D) and exfoliating cells in (E) were enumerated as in $(A)$ and $(C)$, respectively.
A

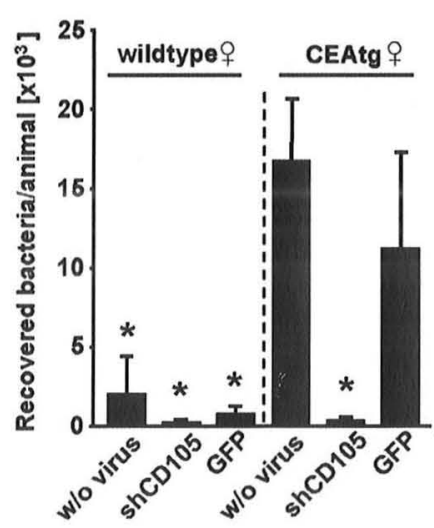

B wildtype

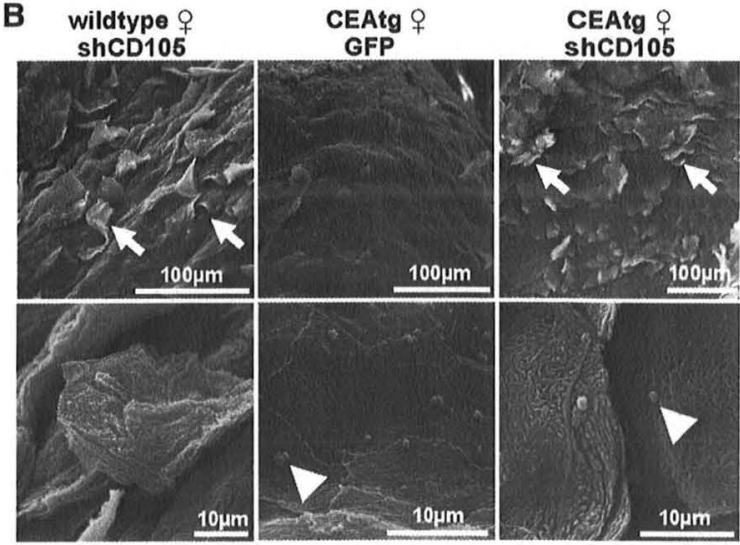

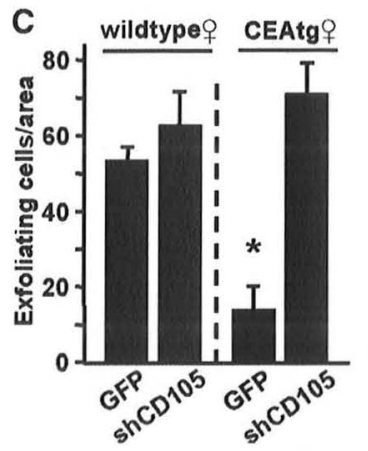
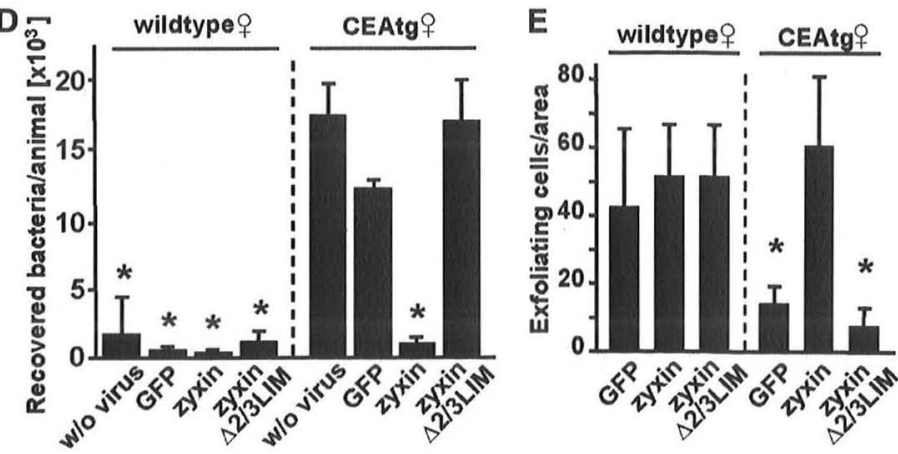
particles driving the expression of zyxin-GFP, zyxin- $\triangle 2 / 3$ LIM-GFP, or GFP, respectively (fig. S11). Delivery of zyxin to the vaginal epithelium did not result in increased exfoliation of the uninfected mucosa (fig. S11). However, zyxin, but not zyxin- $\triangle 2 / 3$ LIM, overexpression blocked the colonization of CEAtg mice by $\mathrm{Opa}_{\mathrm{CEA}}$-expressing gonococci (Fig. 4D). The overexpression of zyxin, but not of zyxin- $\Delta 2 / 3 \mathrm{LIM}$, allowed exfoliation of CEA-positive cells infected with Ngo Opa CEA and reestablished the responsiveness of the vaginal mucosa (Fig. 4E and fig. S11). Thus, CEAinitiated up-regulation of CD105 on superficial epithelial cells and the resulting delocalization of zyxin from integrin-rich focal adhesion sites are the critical molecular events that allow CEACAMbinding microorganisms to counteract the exfoliation response.

Although symptomatic gonococcal infection in humans might be a multistep process orchestrated by additional virulence factors $(13,23)$, our results establish a specific role of Opa $\mathrm{CFA}_{\mathrm{A}}$ proteins in promoting mucosal colonization. In vivo challenge experiments with male volunteers have revealed that after infection of the urethra with non-opaque gonococci, bacteria re-isolated from these volunteers almost invariably converted to an Opa protein-expressing phenotype $(24,25)$. In addition to the urogenital tract, members of the CEACAM family are present on all mucosal surfaces including the nasopharynx (26). These mucosal habitats are colonized by several Gram- negative bacterial species, which make use of unrelated protein adhesins to engage human CEACAMs (27-31). The blockage of epithelial exfoliation afforded by CEACAM binding might have driven this convergent evolution that allows specialized bacteria to transform the mucosa into a dependable platform for colonization.

\section{References and Notes}

1. J. P. Pearson, I. A. Brownlee, in Colonization of Mucosal Surfaces, J. P. Nataro, P. S. Cohen, H. L. T. Mobley, J. N. Weiser, Eds. (ASM Press, Washington, DC, 2005), pp. 3-16.

2. M. A. Mulvey et al., Science 282, 1494 (1998).

3. M. A. Mulvey, ]. D. Schilling, ]. J. Martinez, S. J. Hultgren, Proc. Natl. Acad. Sci. U.S.A. 97, 8829 (2000).

4. I. U. Mysorekar, M. A. Mulvey, S. ]. Hultgren, ]. I. Gordon, J. Biol. Chem. 277, 7412 (2002).

5. P. Muenzner, M. Rohde, S. Kneitz, C. R. Hauck, J. Cell Biol. 170, 825 (2005).

6. M. Kim et al., Nature 459, 578 (2009).

7. WHO, Global Prevalence and Incidence of Selected Curable Sexually Transmitted Infections-Overview and Estimates (World Health Organization, Geneva, 2001).

8. M. A. Melly, C. R. Gregg, Z. A. McGee, J. Infect. Dis. 143, 423 (1981).

9. Z. A. McGee, A. P. Johnson, D. Taylor-Robinson, J. Infect. Dis. 143, 413 (1981).

10. K. F. Tjia, J. P. van Putten, E. Pels, H. C. Zanen, Graefes Arch. Clin. Exp. Ophthalmol. 226, 341 (1988).

11. I. M. Mosleh, H. ]. Boxberger, M. ]. Sessler, T. F. Meyer, Infect. Immun. 65, 3391 (1997).

12. See supporting material on Science Online.

13. M. Virji, Nat. Rev. Microbiol. 7, 274 (2009).

14. M. Voges, V. Bachmann, R. Kammerer, U. Gophna, C. R. Hauck, BMC Microbiol. 10, 117 (2010)

15. C. R. Hauck, T. F. Meyer, Curr. Opin. Microbiol. 6, 43 (2003).
16. A. M. Eades-Perner et al., Cancer Res. 54, 4169 (1994).

17. J. P. van Putten, S. M. Paul, EMBO J. 14, 2144 (1995).

18. T. Chen, R. J. Belland, J. Wilson, J. Swanson, J. Exp. Med 182, 511 (1995).

19. B. A. Conley et al., J. Biol. Chem. 279, 27440 (2004)

20. G. Zambruno et al., J. Cell Biol. 129, 853 (1995).

21. M. Guerrero-Esteo et al., Eur. J. Cell Biol. 78, 614 (1999)

22. L. M. Hoffman et al., J. Cell Biol. 172, 771 (2006).

23. A. ]. Merz, M. So, Annu. Rev. Cell Dev. Biol. 16, 423 (2000).

24. J. Swanson, O. Barrera, ]. Sola, J. Boslego, J. Exp. Med. 168, 2121 (1988).

25. A. E. Jerse et al., J. Exp. Med. 179, 911 (1994).

26. S. Hammarström, Semin. Cancer Biol. 9, 67 (1999).

27. M. Virji, K. Makepeace, D. J. P. Ferguson, S. M. Watt, Mol. Microbiol. 22, 941 (1996).

28. M. Virji et al., Mol. Microbiol. 36, 784 (2000).

29. D. J. Hill, M. Virji, Mol. Microbiol. 48, 117 (2003).

30. C. N. Berger, O. Billker, T. F. Meyer, A. L. Servin, I. Kansau, Mol. Microbiol. 52, 963 (2004).

31. M. Toleman, E. Aho, M. Virji, Cell. Microbiol. 3, 33 (2001).

32. We thank M. C. Beckerle for zyxin-deficient fibroblasts and antibodies; $M$. Chudakov for the mKate CDNA; J. W. Greiner for sending the CEAtg mice; T. F. Meyer fo bacterial strains; D. W. Piston for CDNA of mCerulean; A. J. Schaeffer for hVEC cells; D. Vestweber for providing antibody; C. Hentschel, ]. Scharrer, and R. Mak'anyengo for assistance with SEM; and B. Planitz for expert animal care. Supported by Deutsche Forschungsgemeinschaft grant Ha2856/6-1 (C.R.H.).

\section{Supporting Online Material}

www.sciencemag.org/cgi/content/full/329/5996/1197/DC Materials and Methods

Figs. $\mathbf{S 1}$ to $\mathbf{S 1 0}$

References

14 April 2010; accepted 30 June 2010

10.1126/science.1190892 